\title{
Recherche fondamentale et translationnelle sur le carcinome hépatocellulaire en 2008 : avancées récentes et perspectives
}

Jessica Zucman-Rossi ${ }^{1}$, Bruno Clément ${ }^{2}$, Marie-Annick Buendia ${ }^{3}$, Hervé Lerat ${ }^{4}$, Bernard Van Beers ${ }^{5}$, Pierre Bedossa ${ }^{6}$, Julien Taieb ${ }^{7}$, Jean Rosenbaum ${ }^{8}$

${ }^{1}$ INSERM, U674, Université Paris Diderot - Paris 7, Paris, F-75010 France

${ }^{2}$ INSERM, U620, University de Rennes I, F-35043 Rennes, France

${ }^{3}$ INSERM, U579, Institut Pasteur, Paris, France

${ }^{4}$ INSERM, U841, Département de virologie, Hôpital Henri Mondor, Université Paris 12, France

${ }^{5}$ Service d'imagerie, Hopital Beaujon, AP-HP et INSERM, U772, Paris France

${ }^{6}$ Service d'Anatomie pathologique Hôpital Beaujon, AP-HP, et INSERM, U772, Paris France

${ }^{7}$ Service d'hépatogastro-entérologie et d'oncologie digestive, hôpital européen Georges-Pompidou, Assistance publique-Hôpitaux de Paris, 20, rue Leblanc, 75015 Paris, France

${ }^{8}$ INSERM, U889; Université de Bordeaux, F-33076 Bordeaux, France

Correspondance: Jessica Zucman-Rossi, Inserm U674, Université Paris Diderot - Paris 7, 27 rue Juliette Dodu, Paris, F-75010 France

Titre court : recherche dans le carcinome hépatocellulaire

Mots-clés

carcinome hépatocellulaire, recherche, voie de carcinogenèse, test diagnostiques 


\section{Résumé :}

La carcinogenèse hépatique est en général le résultat d'un processus multiétapes débutant avec l'exposition à différents facteurs de risque; suivi du développement d'une hépatopathie chronique et d'une cirrhose, véritable lésion prénéoplasique; et pour finir après le développement carcinome hépatocellulaire, il existe de nombreux évènements qui vont controler l'agressivité de ces tumeurs. Le but de ce travail a été d'identifier les forces et priorités de la recherche fondamentale et translationnelle dans le domaine de la carcinogenèse hépatique dans son contexte international.

Mots-clés : carcinome hépatocellulaire, recherche, voie de carcinogenèse, test diagnostiques 


\section{Abstract}

Hepatocellular carcinogenesis is usually the result of a muti-step process. It begin with an exposure to various risk factors; followed by the development of a chronic hepatitis and cirrhosis that is a pre-neoplastic step; and finally after the occurrence of an hepatocellular carcinoma (HCC), different molecular events control aggressiveness of the tumors. The aim of this work was to identify in the international context, forces and priorities of the fundamental and translational HCC research.

Key words: Hepatocellular carcinoma, fundamental research, translational research, carcinogenesis pathway, diagnostic test 


\section{Carcinogenèse hépatique.}

Le $\mathrm{CHC}$ est probablement l'une des tumeurs dont on connaît le mieux les agents étiologiques[1]. II s'agit principalement de l'infection chronique par les virus de l'hépatite $B$ et $C$ (VHB et $\mathrm{VHC}$ ), l'exposition à l'Aflatoxine B1, la consommation excessive d'alcool, les maladies génétiques métaboliques comme l'hémochromatose ou le déficit en alpha1 anti-trypsine. Malgré cela, de très nombreuses études n'ont conduit qu'à des données incomplètes quant aux mécanismes initiateurs de l'hépatocarcinogenèse. En effet, le caractère multifactoriel de ces mécanismes en rend la compréhension complexe et peu de gènes de prédisposition ont été décrits pour ces tumeurs (voir pour revue, [2]). Cependant, l'existence d'agrégations familiales de cas de $\mathrm{CHC}$ lié à l'infection virale $\mathrm{B}$, le potentiel carcinogène de l'Aflatoxine B1 qui varie en fonction des polymorphismes individuels pour la détoxification du métabolite mutagène époxide 8,9 de l'aflatoxine et l'existence de polymorphismes génétiques associés au développement de $\mathrm{CHC}$ chez les malades présentant une cirrhose d'origine alcoolique [3,4] soulignent l'importance des interactions du type "gènes-environnement" dans les étapes précoces de la carcinogenèse hépatique.

La carcinogenèse hépatique est dans la très grande majorité des cas un processus multi-étapes. L'hépatite chronique et la cirrhose qui précèdent dans plus de $90 \%$ des cas l'apparition de $\mathrm{CHC}$ sont de véritables étapes pré-néoplasiques. En effet, ces lésions génèrent une inflammation associée à une régénération hépatocytaire qui favorisent le stress oxydatif, la sénescence réplicative et l'accumulation d'altérations génétiques et épigénétiques dans les hépatocytes [5]. Ainsi, les nodules cirrhotiques dysplasiques de haut grade sont de véritables lésions pré-néoplasiques fréquemment monoclonales, présentant des aberrations chromosomiques et la réexpression de la télomérase qui participe à l'immortalisation des hépatocytes [6]. Cependant, les altérations structurales des gènes impliqués dans le contrôle de la prolifération cellulaire, c'est à dire les mutations activatrices d'oncogènes ou les mutations inactivatrices de gènes suppresseurs de tumeurs, n'ont jamais été identifiées jusqu'à présent dans les nodules cirrhotiques alors qu'elles sont très fréquemment accumulées dans les $\mathrm{CHC}$.

La diversité des facteurs d'initiation de la carcinogenèse dont un grand nombre sont spécifiques d'un facteur de risque donné explique en grande partie la 
complexité des mécanismes de carcinogenèse mis en jeu et sélectionnés dans les cellules tumorales. La compréhension de cette complexité nous a permis de faire des progrès considérables en sortant du modèle simpliste qui était encore d'actualité dans les années 90 pour la plupart des tumeurs : " un facteur de risque - un gène altéré - une voie de signalisation - un traitement ». En effet, ces dernières années, la recherche dans le domaine du $\mathrm{CHC}$ a fait des progrès à la fois sur le plan fondamental et translationnel et ce sur plusieurs aspects qui pourront déboucher dans un proche futur sur des applications cliniques pratiques (Figure 1).

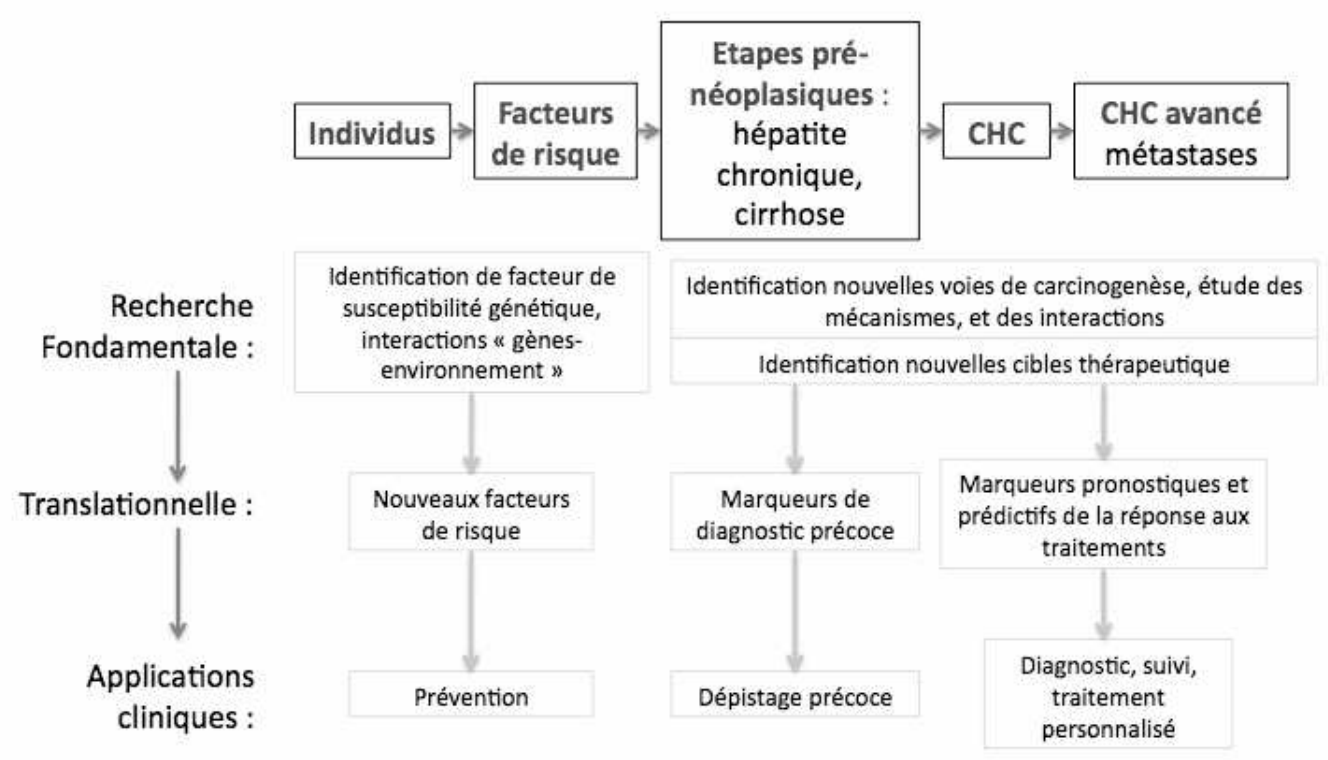

Figure 1: Grands axes de recherche dans le domaine du carcinome hépatocellulaire

1- Les principales avancées récentes dans la compréhension des mécanismes de carcinogenèse hépatique 
Dans ce domaine, le développement de nouveaux d'outils d'étude comme les souris génétiquement modifiées, les puces à ADN, l'analyse du protéome a été prépondérant pour faire progresser la recherche sur le $\mathrm{CHC}$. Parmi les progrès spectaculaires qui ont été faits on peut citer 5 grands domaines :

\section{a- Carcinogenèse induite par le virus de l'hépatite $B$}

Des découvertes importantes ont été réalisées en France il y a une vingtaine d'années et ont permis d'identifier les premiers oncogènes ciblés par l'infection virale B chez l'homme et la marmotte $[7,8,9,10,11]$. Ensuite de nombreux travaux fondamentaux et cliniques ont contribué à faire progresser nos connaissances dans le domaine de la carcinogenèse liée au virus de l'hépatite $B[12,13]$. En particulier, l'étude des sites d'insertion du VHB dans le génome des cellules tumorales chez l'homme a permis d'identifier un grand nombre de gènes potentiellement importants pour la tumorigenèse comme ceux de la télomérase, ou de MLL qui sont ciblés de manière récurrente par le VHB $[13,14]$. L'analyse du rôle d'HBV dans la carcinogenèse a aussi bénéficié de la construction de plusieurs modèles animaux génétiquement modifiés et de nombreuses études de la fonction des protéines virales dans le contrôle de la prolifération cellulaire [15, 16, 17 ].

\section{b- Carcinogenèse induite par le virus de l'hépatite $C$}

Concernant les mécanismes de carcinogenèse induite par le VHC, le niveau de connaissance est moins avancé que pour le VHB car il plus difficile d'identifier des mécanismes directement liés et spécifiques du virus lui-même [18]. Un modèle d'étude particulièrement intéressant pour étudier ces mécanismes est celui des souris transgéniques avec l'ensemble de la séquence codante d'HCV qui développent des carcinomes hépatocellulaires [19, 20].

\section{c- Identification de nouvelles voies de carcinogenèse}

De nombreuses voies de signalisation ont été identifiées comme altérées dans les carcinomes hépatocellulaires. Deux d'entres elles sont fréquemment altérées dans les $\mathrm{CHC}$ chez l'homme. II s'agit premièrement de la voie Wnt ß-caténine activée par mutation activatrice de la ß-caténine dans environ 20 à $40 \%$ des cas [21, 22] et 
deuxièmement de l'inactivation de la voie TP53 dans 20 à $50 \%$ des $\mathrm{CHC}$ avec chez les malades exposés à l'Aflatoxine B1 une mutation spécifique au codon 249 [23, 24]. D'autres gènes peuvent contribuer à l'activation de la voie WNT/ß-caténine comme les axines 1 et 2 , et de nombreuses autres voies de signalisation (Ras, TGFß, MET, IGF, AKT...) ont été identifiées (voir pour revue, [2, 25]). Plus récemment, le rôle des altérations épigénétiques (méthylation par exemple) et des protéines jouant un rôle dans le remodelage de la chromatine (comme la reptine) a été identifié dans de nombreux cas de carcinomes hépatocellulaires [26, 27]. Un des enjeux importants actuellement est d'une part de compléter le répertoire des voies de carcinogenèse altérées et de comprendre par quels mécanismes ces voies coopèrent entre elles.

\section{d- Etude du microenvironnement tumoral}

Plusieurs mécanismes moléculaires ont été décrits comme responsables de la dissémination cellulaire, de l'invasion et des métastases. En particulier, c'est le cas de la dérégulation de nm23, ostéopontine, Rho C, MMP2, MMP9, MMP14 ou TGFß [28, 29]. Par ailleurs l'importance du microenvironnement tumoral a aussi été identifiée par la mise en évidence dans le tissu hépatique adjacent à la tumeur d'une signature de type interleukine corrélée à l'envahissement veineux et au développement de métastases et par le rôle des myofibroblastes et du remodelage de la matrice extracellulaire dans l'invasion tumorale [30, 31]. Par ailleurs, l'identification récente du rôle de la matrice extra-cellulaire, et plus particulièrement du variant V3 du collagène 18, dans sa capacité à inhiber la voie WNT est une découverte récente qui pointe une nouvelle fois le rôle du microenvironnement tumoral dans le contrôle de la croissance tumorale [32]. Enfin, surtout dans le contexte d'infection virale, le système immunitaire joue un rôle important dans le contrôle de la prolifération hépatocytaire et en particulier la réponse des lymphocyte $\mathrm{T}$ (effectrice et régulatrice) [33].Actuellement, il n'existe pas de modèle murin permettant de modéliser de la manière satisfaisante la séquence de carcinogenèse foie sain => cirrhose $=>$ développement de $\mathrm{CHC}$ en présence ou en absence de séquences virales. C'est probablement un enjeu majeur qui permettra de mieux comprendre le rôle de l'environnement tumoral à la fois dans les étapes précoces et plus tardives de la carcinogenèse. 


\section{e- Classification moléculaire des $\mathrm{CHC}$}

Les analyses du transcriptome de grandes séries de $\mathrm{CHC}$ ont permis récemment d'identifier une véritable classification des ces tumeurs chez l'homme. II est tout à fait remarquable de noter que cette classification est aussi retrouvée dans les tumeurs murines. Les points importants de cette classification sont: l'identification d'un sous-groupe de tumeur de type "hépatoblast-like» [34], l'étroite relation existant entre les différents sous-groupes moléculaires avec les facteurs de risque [35, 36-38], les altérations génétiques et épigénétiques [26, 35], les anomalies d'expression des microARN [39], le phénotype tumoral [40] et le pronostic des malades. Ces classifications devront être affinées dans le futur et elles permettront de mieux comprendre les interactions entre les différentes voies de carcinogenèse. Elles constituent des outils de choix pour l'identification de nouveaux ciblages thérapeutiques raisonnés.

2- Les nouvelles perspectives dans le domaine de la recherche translationnelle sur le carcinome hépatocellulaire.

La recherche translationnelle en cancérologie a un rôle très important pour favoriser le passage de la recherche fondamentale vers les applications cliniques. Le but de cette recherche est par exemple d'identifier et de valider les meilleurs marqueurs qui auront un intérêt dans la prise en charge des malades. II s'agit donc d'une étape indispensable pour la mise en application de découvertes scientifiques. Dans ce cadre, les collections de ressources biologiques sont particulièrement importantes pour la réussite des projets. En France, nous bénéficions du réseau national de collecte d'échantillons tumoraux qui dispose d'une base de données centralisée et de protocoles de standardisation des procédures de collecte et d'annotation (http://chc.isped.u-bordeaux2.fr/). Plusieurs axes de recherche translationnelle sont actuellement en cours de développement rapide, et parmi eux deux sont particulièrement prometteurs.

\section{a- Identification de marqueurs précoces de survenue des $\mathrm{CHC}$}

Ce secteur de recherche est essentiel en raison de ses débouchés à court et moyen termes pour la surveillance et la prise en charge thérapeutique des malades cirrhotiques. II est étroitement lié aux cohortes de malades cirrhotiques surveillés 
pour la survenue de tumeurs dans le cadre d'infection virale $C$ (cohorte ANRS CIRVIR) ou en dehors d'infection virale. Les études en particulier de protéomique qui ont déjà abouti à l'identification de nouveaux marqueurs [41, 42] pourront être étendues et validées grâce en particulier aux biobanques qui sont en cours de constitution en France. Dans le même cadre, les techniques d'imagerie fonctionnelle et moléculaires ont fait d'important progrès ces dernières années et elles ouvrent des perspectives intéressantes pour le diagnostic précoce et la caractérisation des nodules hépatiques $[43,44]$, ainsi que pour l'évaluation de la réponse aux traitements.

\section{b- Etude de la prédiction de la réponse ou de la résistance aux traitements}

Le développement récent de thérapies ciblées dont une au moins (Sorafenib) a déjà démontré son efficacité en terme de survie dans le traitement du $\mathrm{CHC}$ pose la question importante de l'identification des malades qui bénéficieront de ces nouveaux traitements [45]. Cette question se pose de manière similaire pour affiner les indications des traitements curatifs classiques chirurgicaux ou par radiofréquence [46]. Plusieurs marqueurs pronostiques ont déjà été identifiés par le biais d'analyses histologiques, génétiques, chromosomiques, transcriptomiques, protéomique [34, 35, 47, 48]. Actuellement, les validations multicentriques de ces marqueurs sont essentielles à faire pour identifier les plus robustes tout en tenant compte de la diversité des tumeurs. Enfin, la recherche d'efficacité de différentes drogues bénéficie des nombreux modèles murins de $\mathrm{CHC}$ développés ces dernières années mais aussi des modèles cellulaires utilisables in vitro pour effectuer des tests de candidats médicaments (toxicité, métabolisme...).

\section{Conclusions}

Le développement de la recherche fondamentale et translationnelle dans le domaine du $\mathrm{CHC}$ a été important ces 20 dernières années et les équipes françaises ont eu un rôle de tout premier rang sur le plan international. Globalement la recherche sur le $\mathrm{CHC}$ en France pourrait aussi bénéficier d'une meilleure interaction multidisciplinaire. Il y a notamment un intérêt certain à améliorer les interactions entre recherche fondamentale et recherche clinique pour que les avancées scientifiques puissent plus rapidement bénéficier aux malades en améliorant la prévention et le 
diagnostic mais aussi pour se diriger vers une prise en charge thérapeutique personnalisée.

\section{Remerciements}

Ce travail de synthèse s'est déroulé dans le cadre du Programme d'Actions Intégrées de Recherche sur le Carcinome HépatoCellulaire (PAIR-CHC) co-financé par l'Inca, l'ARC et l'ANRS. Nous remercions particulièrement Raymond Pamphile, Claire Verbeke, Dominique Franco et Fabien Calvo pour leur aide précieuse.

\section{Références}

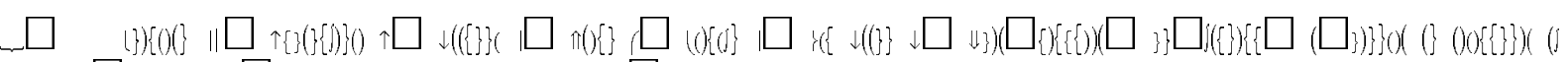

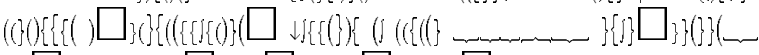

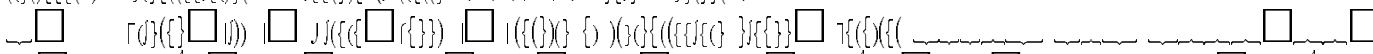

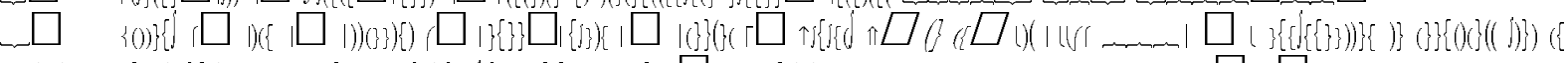

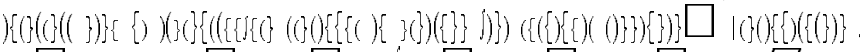

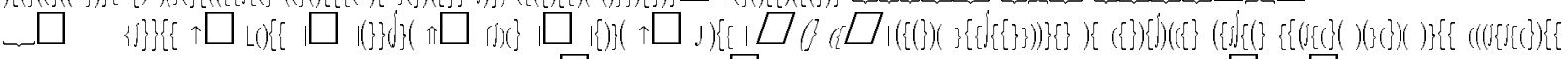

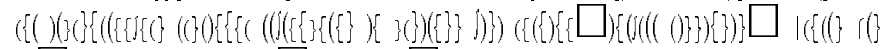

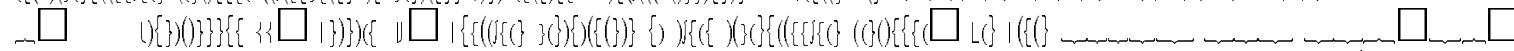

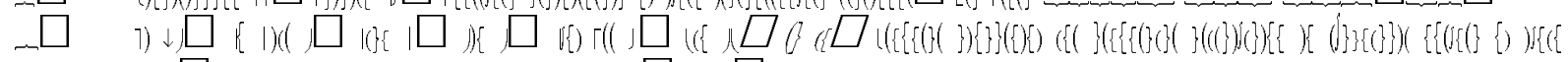

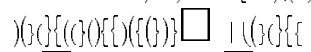

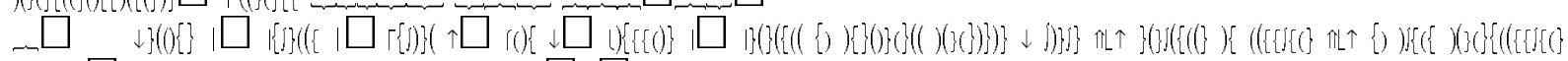
(c) $\{\{\{[\square<\}\}\}$

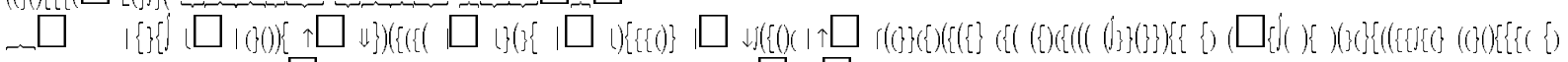

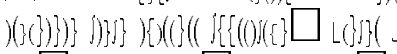

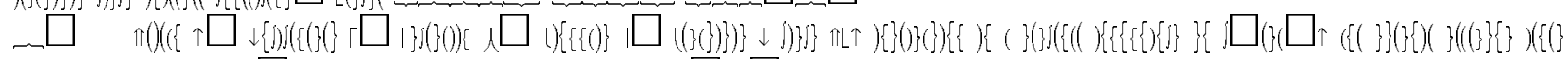

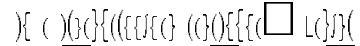

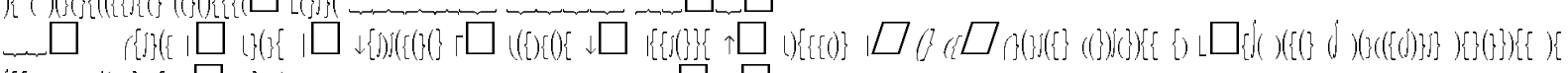

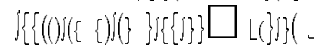

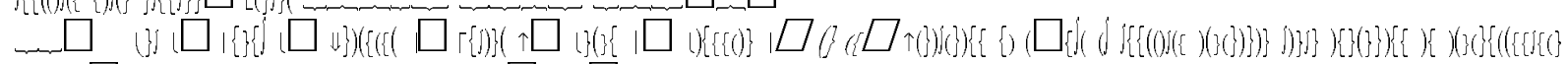

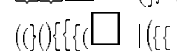

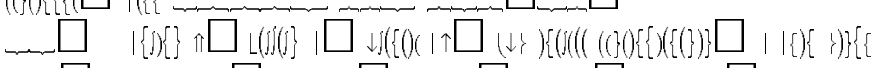

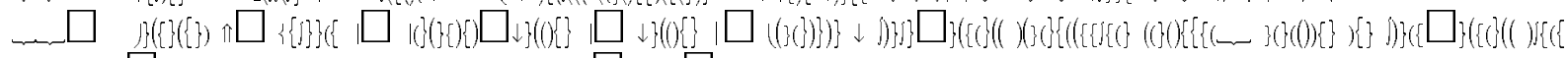

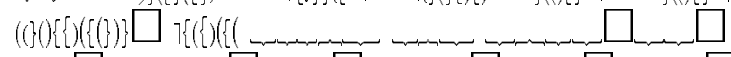

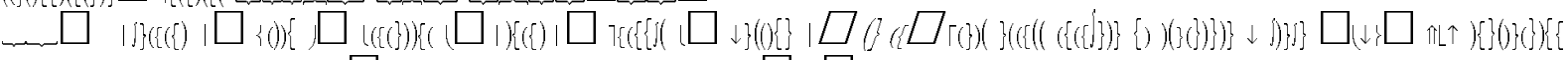

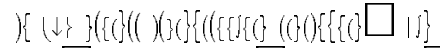

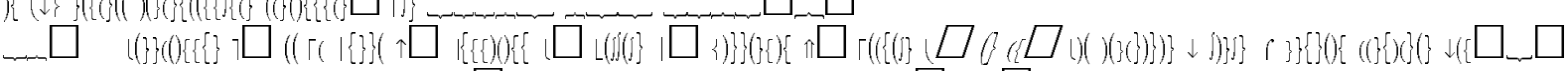

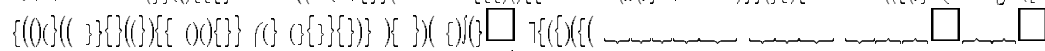

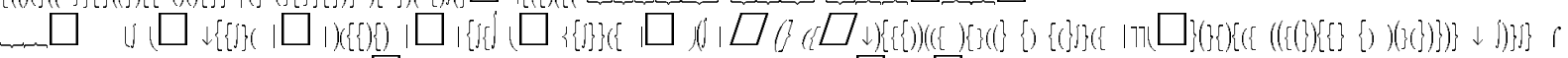

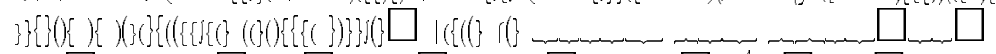

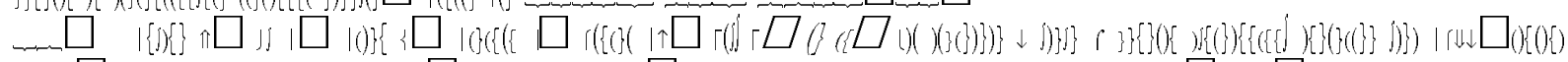

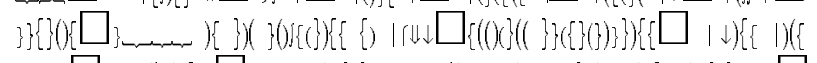

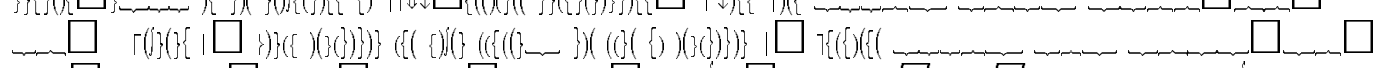

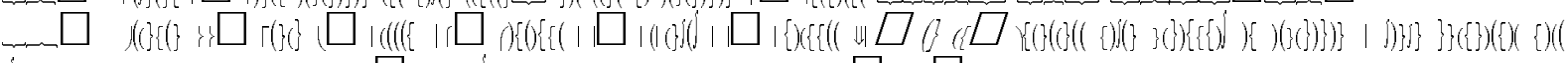

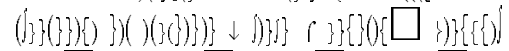

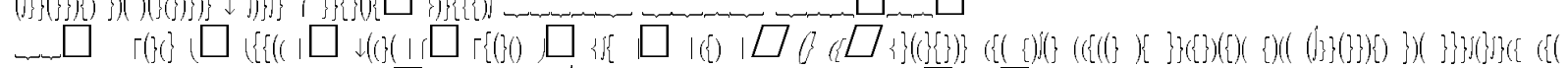

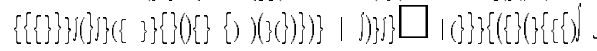




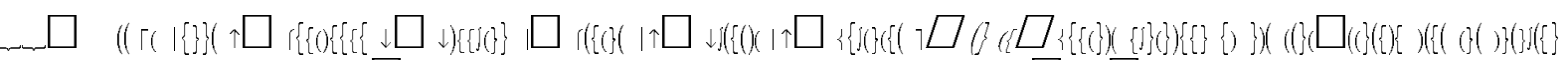

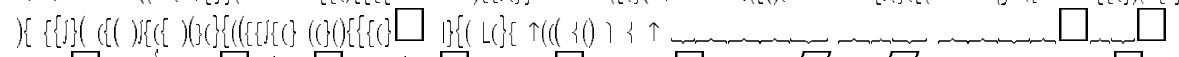

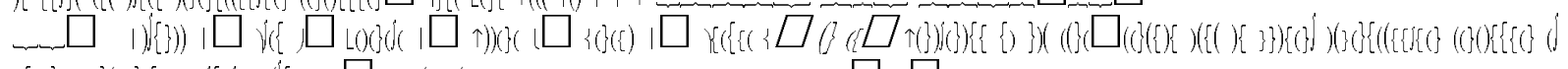

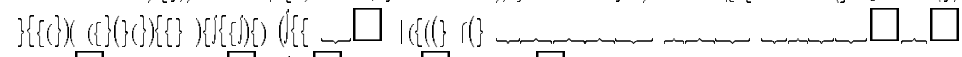

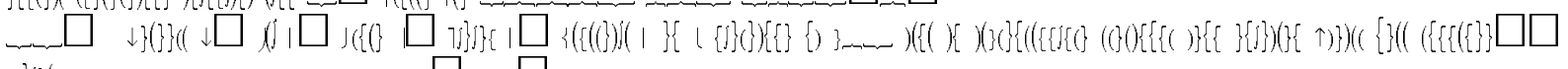
L(3)乃 (

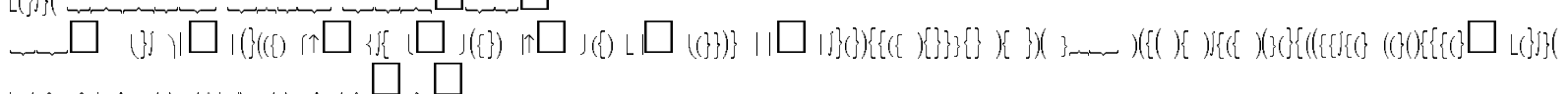

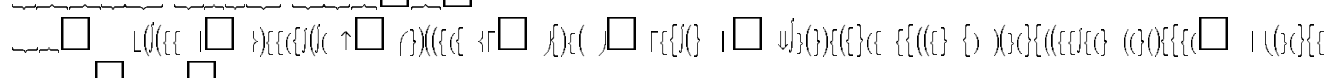
$\square$

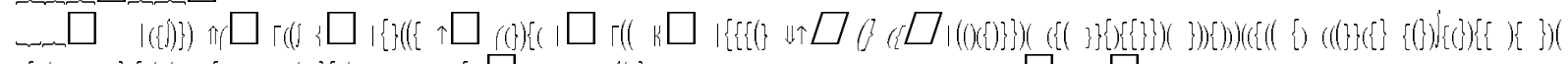

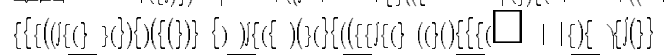

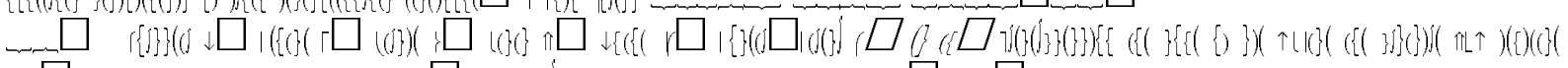

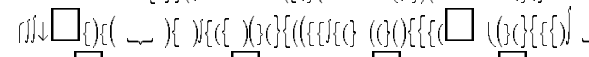

(a)

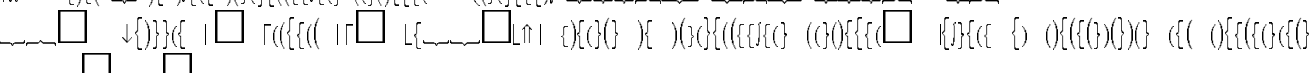
$\square+\square$

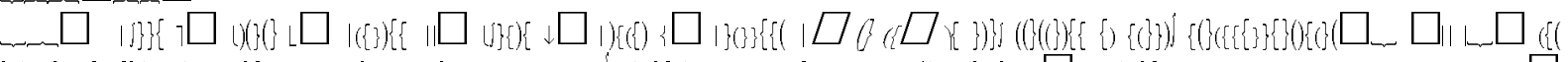

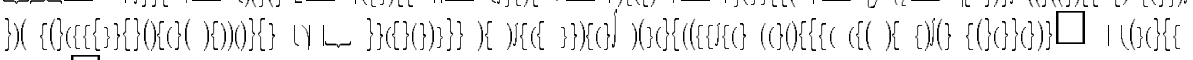

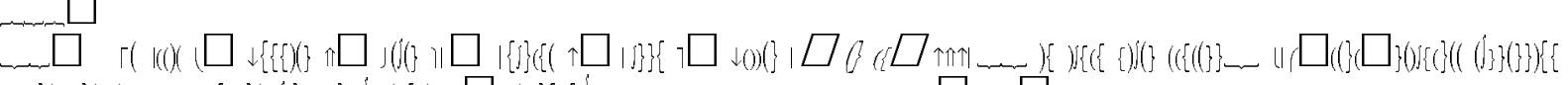

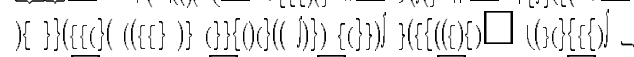

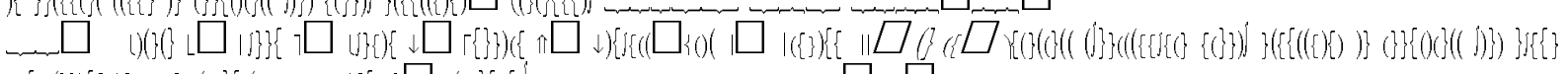

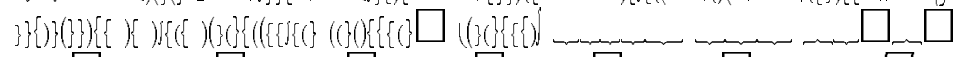

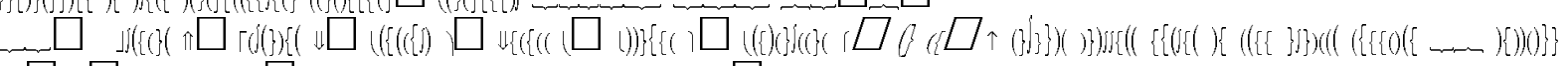

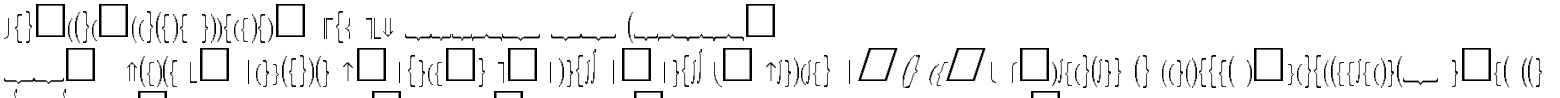

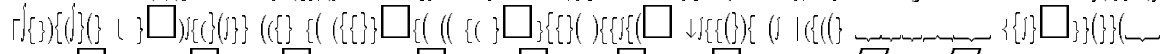

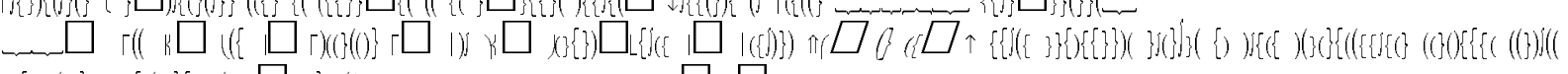

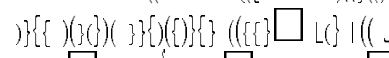

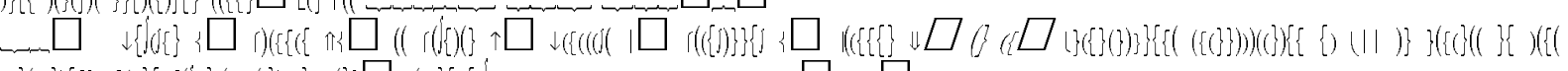

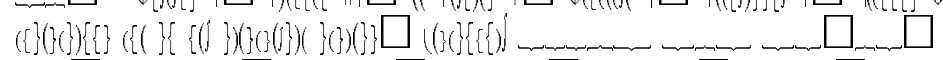

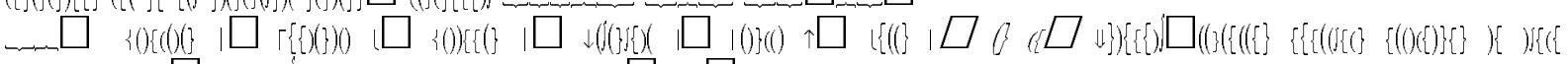

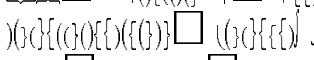

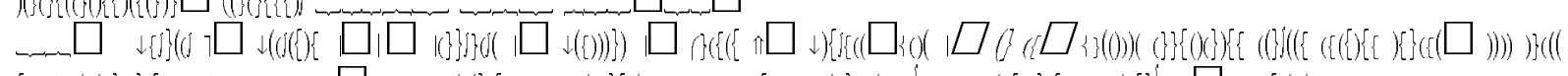

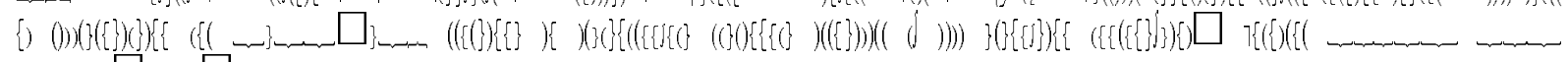
$\square$

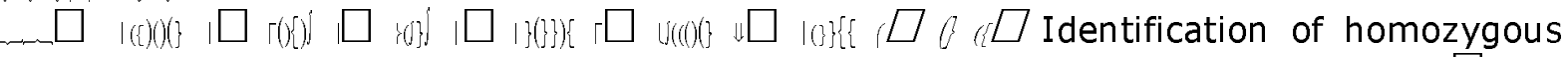
deletions at chromosome 16q23 in Aflatoxin B1 exposed hepatocellular carcinoma $\square$ Ti(f) $(2)$

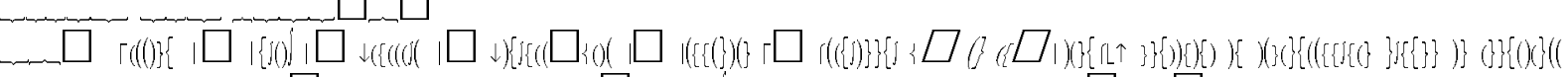

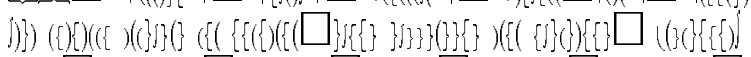

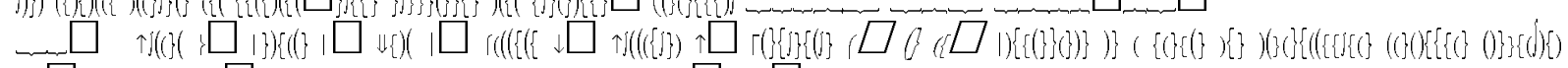
(3) $\square(3\}\{(2)\{\{\}\}\}\{\{\{\} \square \mid k\}\{\{$

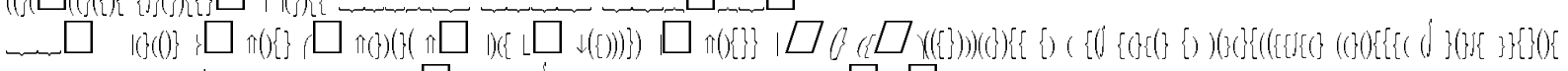

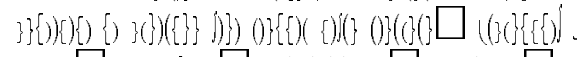

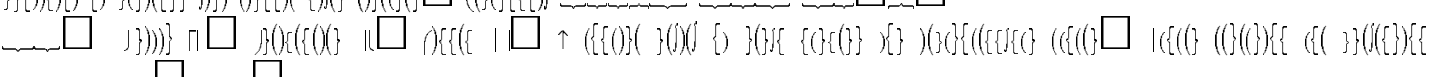

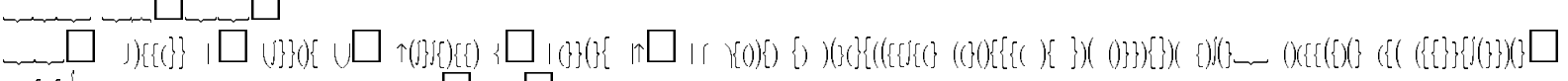
(io) $\{\{x\}$

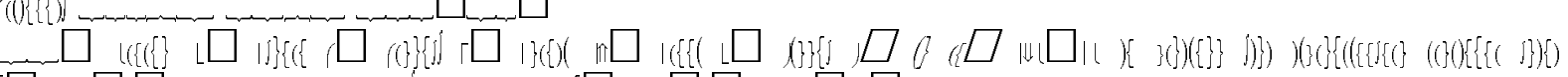

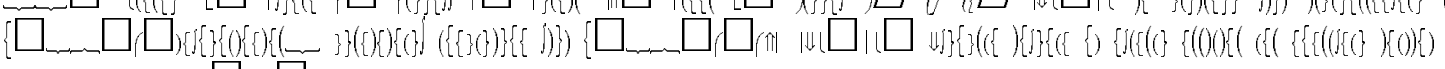
$+\square$

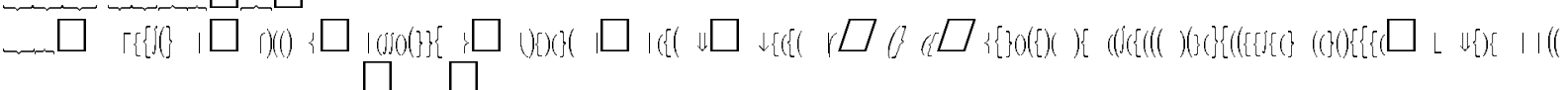




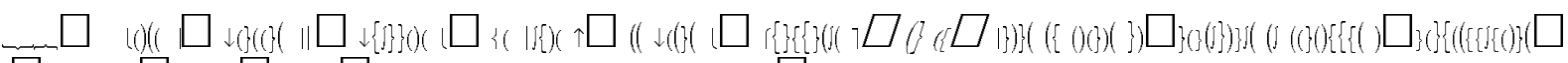

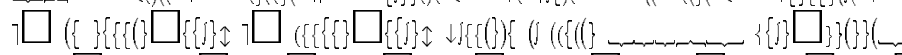

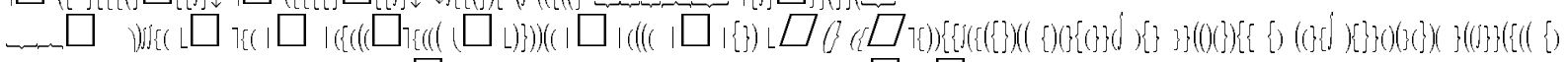

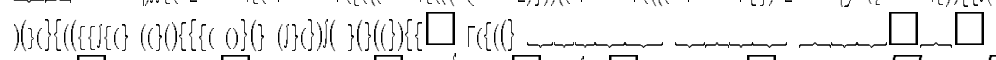

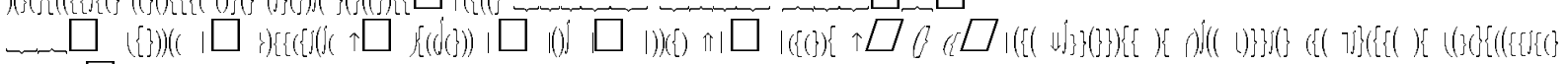

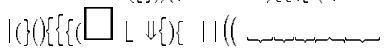

